\title{
A Study on the Mechanism of Social Robot Attitude Formation through Consumer Gaze Analysis: Focusing on the Robot Face
}

\author{
Sangjip $\mathrm{Ha}^{1}$, Eun-ju $\mathrm{Yi}^{1}$, In-jin Yoo ${ }^{1}$, and Do-Hyung Park ${ }^{1, *}$ \\ ${ }^{1}$ Graduate School of Business IT, Kookmin University, Seoul, The Republic of Korea
}

\begin{abstract}
This study intends to utilize eye tracking for the appearance of a robot, which is one of the trends in social robot design research. We suggest a research model with the entire stage from the consumer gaze response to the perceived consumer beliefs and further their attitudes toward social robots. Specifically, the eye tracking indicators used in this study are Fixation, First Visit, Total Viewed Stay Time, and Number of Revisits. Also, Areas of Interest are selected to the face, eyes, lips, and full-body of a social robot. In the first relationship, we check which element of the social robot design the consumer's gaze stays on, and how the gaze on each element affects consumer beliefs. The consumer beliefs are considered as the social robot's emotional expression, humanness, and facial prominence. Second, we explore whether the formation of consumer attitudes is possible through two major channels. One is the path that the consumer beliefs formed through the gaze influence their attitude, and the other is the path that the consumer gaze response directly influences the attitude. This study made a theoretical contribution in that it finally analysed the path of consumer attitude formation from various angles by linking the gaze tracking reaction and consumer perception. In addition, it is expected to make practical contributions in the suggestion of specific design insights that can be used as a reference for designing social robots.
\end{abstract}

Keywords: Social Robot, Gaze Analysis, Eye Tracking, Robot Design, Attitude Formation

\section{Introduction}

Robot is coming to the life of human. Robots initially being installed in the factory for industrial use are being transformed for personal use and spread to the daily life of diverse users. Researchers are investigating how the life with robots would influence to human being from various perspective and background. Personal Robot is largely divided into two: One is Service Robot, which is a type of tools for movement, maintenance, cleaning etc. and the other is Social Robot, which is distinguished from that of former in that it is regarded as a companion or family for its main purpose is to communicate at a high level including emotional exchange [1].

\footnotetext{
* Corresponding author: dohyungpark@,kookmin.ac.kr
} 
Research on Social Robots are specifying them as Social Agent or Social Entity able to move autonomously and verbal conversation and focusing on the interaction between the users and robots (Human-Robot Interaction: HRI). More specifically, studies on social robot design are classified as surface and appearance design, functional and role-based design, and interaction design including verbal-nonverbal expression.

Social robots, which are destined to support human's well-being, are equipped with face, body, leg(s), and arm(s) for better physical help by moving like human and easier interaction by showing their emotion and intends like that of human. Certainly, people showed intimacy and stronger anthropomorphism as robot's appearance gets closer to human. However, researchers also found out that this preference stops and decline dramatically at a certain level for people experienced unpleasant, repulsion and abhorrence [2]. This phenomenon called Uncanny Valley represents that the appearance of robots is one of the key elements in designing robots for it has great impact on people's attitude. Therefore, designing the robot's appearance needs new approach which is not merely drawing the external features but regard it as one of the key factors in determining user's attitude.

To elucidate the mechanism of use's attitude on social robot, we designed an experiment of looking at the picture of social robots on screen, collected the user's gaze tracking data with eye-tracker and surveyed to ask about the attitude to each robot. The tracking indicators used for this study is the number (of times) of the fixed view (Fixation), time (in seconds) to the first visit (First Visit), duration (in seconds) of the totally stayed (Total Viewed), and number (of times) of the visits (Revisits). Segmented AOI (Areas of Interests) is face, eyes, lips and fullbody. Collected data from survey is design evaluation on each social robot's emotional expression, humanness, and facial prominence and attitude as a dependent variable.

The theoretical contribution of this research is that we have connected 2 different data between gaze tracking reaction and consumer perception and finally analysed the path of consumer attitude formation with diverse perspective. The result of this research provides specific design guideline in designing the social robot at work.

\section{Theoretical backgrounds}

\subsection{Social Robot}

The first robot materialized by scientific technology was installed and fixed on the automobile assembly line to replace humans' simple and repeatable labour force. Nowadays we can find robots are at home, in room and on desk. Unlike industrial robots, they were born to serve person [1]. They work either as a type of material equipment in charge of maintenance, cleaning or as a type of companion or as a type of companion or family members in charge if safe, entertainment and emotional well-being. The latter type of robot is called as Social Robot, which is capable in verbal or non-verbal communication and interact as humans do $[3,4]$. The qualification of social robot mentioned by prior studies is that it has the proper embodiment for its use, the feeling of living creature, face recognition function, its own reaction to each situation and motion that is designed to be easily understood by users [1]. Also studies on the guideline in designing social robots suggest that it gratifies the user's expectation, communicate verbally or non-verbally and comply with human's social norms [5].

Meanwhile as conceptual social robots become invented and more and more social robots are being commercialized out of laboratory, designing has become one of the most significant tasks. Nowadays designing means not only drawing visual elements but also creating functional factors. Specifically, the former is modelling, formative and artistic area and applied in producing the surface of robot. The latter is related with functional planning to 
fulfil user's purpose: planning itself, planning process and status that the planned elements are installed [6].

\subsection{Social Robot Design}

We classified 3 types of research in social robot design. First is the surface design, which is in artistic area and deals with human-likeness and aesthetic balance of the robot's face, body, $\operatorname{arm}(\mathrm{s})$, and $\operatorname{leg}(\mathrm{s})$. Most representative jargon in this field is the 'Uncanny Valley' phenomenon which means that the preference for a robot increases as robot's human likeness increases until it reaches at a certain likeness level and paradoxically falls significantly down after the certain likeness level [2].

Second is the functional and role-based design to meet user's needs. Social robot may have its unique and special tasks as intended for example elderly care [7], autistic child screening [8] and therapy [9-a], children education [9], and so on. Also, it is designed to function in the emotional areas as a robot counsellor and social areas to be a good member in the Human-robot-team.

Finally, third field is the designing the engagement between robot and users. Social robot has diverse means to induce interaction with users. It fits out with both verbal and non-verbal communication including facial expression, body motion, effect sound. Unlike the chatbot service which allows text or tags only or AI speakers which allows sounds only, robot has its presence, and this embodiment increments user's social benefit a lot [11]. It may dance or hug for user's pleasure and take or lift user' body in accordance with user's wish. Researchers have commented the elements to consider for a social robot to improve user's gratification such as softness, size, temperature, movement speed, pressure, voice-tone (Friend-like vs. Engineer-like), voice pitch and nudging $[12,13]$.

\subsection{The Effect of Social Robot Design on Consumer Attitudes}

There is a difference between industrial robots and social robots. While the appearance design of industrial robots emphasizes the performance and functional aspects of robots, social robots emphasize the cognitive and emotional exchanges through interaction with humans [14]. Furthermore, the appearance design of such social robots affects consumers, and consumers may change their evaluations and attitudes toward robots [15]. Therefore, when considering social robot design elements, individual perception or psychological characteristics should not be overlooked.

As representative factors, "Robot face", "anthropomorphism", and "emotional expression" affect the attitude of consumers. First, the face design of social robots is used as a means of basic communication with humans, and it can also affect the attitude of consumers [16]. For example, in previous studies, preference for the appearance of social robots differs depending on the proportion and type of face, and there is also a study that reported that the more a humanlike face is designed, the greater the preference of consumers [17].

Second, it is a design about the anthropomorphism of social robots. anthropomorphism refers to giving human characteristics to non-human objects such as animals and inanimate objects [14]. In particular, the anthropomorphism trend can be mainly confirmed in robots that require human-like shapes and functions, is applied in various ways in relation to physical shape, motion, and interaction when considering robot design [18]. This level of anthropomorphism affects consumer preferences. For example, it has been found that consumers expect robots used in public services to behave similarly to actual service workers, and the more the verbal interaction is similar to humans, the more consumers prefer [19]. 
Third, it is a design related to the emotional expression of social robots. For the expression of social robots, non-verbal expressions such as gestures, facial expressions, intonations, gaze directions, and postures are used for effective interaction with users [20]. In particular, when expressing emotions through robot movements, consumers may perceive that the robot has emotions. In other words, non-verbal expressions of robots play an important role in forming social relationships with robots [21]. Furthermore, in a study that considered the emotional expression and expression of the robot, the evaluation of consumers was different depending on the shape of the pupil, which is an important medium for conveying emotions [22].

In previous studies, the factors that can influence the consumer's evaluation of social robot design were subdivided and the influence of each factor was explored. However, these studies have limitations in the objectivity of the methodology because they were conducted based on users' subjective questionnaires and interviews. In other words, objective responses of consumers to social robot design were not collected, and precise measurement tools were not used. Therefore, in this study, the factors affecting the attitude of consumers and the mechanism of forming the attitude are to be verified using objective indicators measured by eye tracking.

\subsection{Eye Tracking}

Eye tracking identifies the point where a person's gaze has stayed, and it refers to the process of measuring the relative movement of the eyes based on the position of the head This movement of the gaze can be explored through the eye tracking device, and it can be observed by visualizing it based on the data derived through the eye tracking. In particular, research has been mainly conducted to designate an Area of Interest (AOI) and to explore where the focus is mainly on among the areas of interest. Eye tracking is being used in various fields, among which there is a study related to social robots. First, a study that confirmed the interaction between a robot character and a person report that the human gaze has a similar pattern when dealing with a robot and a real person [23]. In other words, when a person interacts with a robot, the result is that the gaze stays in the mouth for a long time as when interacting with a person. Meanwhile, to study the design of the robot, an eye tracking device and an EEG device were also used. As a result of the study measured through two devices, it was reported that the features of the head and face were more important than the body organs such as arms and legs of the robot in the appearance of the robot [24].

Meanwhile, eye tracking utilizes various measuring indicators in research. In this study, we tried to use the indicators that are mainly used among them. The first is the 'fixation', which is information about how long the user's gaze stayed on the object. Fixation is usually the most important indicator used because it is possible to confirm which specific area the user is watching, and to objectively explore the user's attention or interest [25]. The fixation collected by the eye tracking device can be subdivided into fixed point coordinates as well as fixed time, changed time, total time the gaze stayed, the distance from the previous fixed point, and movement speed.

The second is the gaze path. It is an indicator of the overall pattern of gaze movement on the object. The gaze path has the advantage of informing a comprehensive gaze movement pattern as the path the gaze has moved is visualized and expressed according to time sequence. In particular, the gaze path is mainly used in design fields such as marketing, product, and outdoor advertising [26].

In summary, in this study, the fixation and the gaze path were subdivided and collected with an eye tracking device. "Fixation" intends to utilize "Number of the fixed view (Fixation)", "Time (in seconds) to the first visit (First Visit)", and "Duration (in second) of the totally stayed (Total Viewed)". In addition, we want to utilize the "Number of the revisits(revisits)", which is data reconstructed to reflect "gaze path" as a quantitative indicator. 


\subsection{Attitude Formation Mechanism Based on Consumer Gaze Response}

This study intends to analyse it by composing it as one research model. This study deals with the relationship between consumer gaze response, perceived beliefs about social robots, and attitudes toward social robots. Specifically, first, it checks which element of the social robot design the consumer's gaze stays on object, and how the gaze on each element affects the consumer's beliefs. Since the face of the social robot has been treated as important in the previous research, we would like to divide the face of the social robot into eyes and mouth in detail and compare it with the whole body. The consumer's beliefs considered the social robot's emotional expression (Expression), humanness (Human-Like), and facial prominence (Facial-Highlighted). Second, we want to check whether it is possible to form a consumer's attitude through two main ways. One is the way in which the beliefs of consumers formed through the gaze influence the attitude, and the other is the direct way in which the gaze of the consumer affects the attitude. Instead of considering all beliefs about social robots, this study tried to confirm the influence of beliefs not covered in this study through the direct influence path of the gaze. The research model proposed in this study is summarized and expressed in $<$ Figure $1>$.

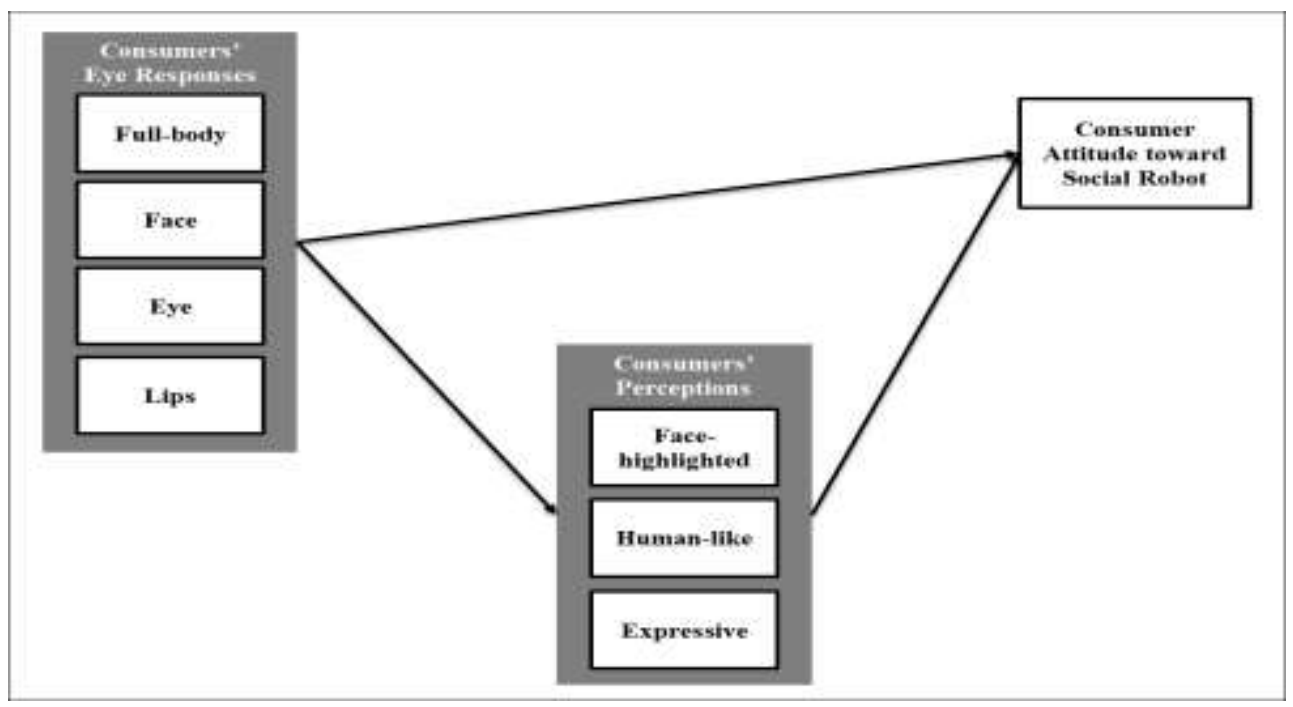

Fig. 1. Research Model Design

Source: Own processing.

\section{Research process}

\subsection{Experiment Participant}

This study was conducted with undergraduate and graduate students participating in UROP (Undergraduate Research Opportunities Program) classes at K University in Seoul. A total of 32 participants were recruited and respectively consisted of 17 males $(53.1 \%)$ and 15 females $(46.6 \%)$, ensuring a relatively equal sex ratio between men and women. The ages of the participants respectively ranged from their 20 s to 40 s, with $94 \%$ in their 20 s, $3 \%$ in their $30 \mathrm{~s}$, and $3 \%$ in their $40 \mathrm{~s}$, with an average age of 25.78 . 


\subsection{Measurement Tool}

The eye tracking measurement tool was measured using Gazepoint's 'GP3' device. The device recognized the pupil shape of the subject through the infrared sensor of the camera and recorded the central position of the pupil as a $60 \mathrm{~Hz}$ gaze response per second through image analysis software. The GP3 device was placed under the monitor of the computer where the subject was outputting the stimulus, and the subject's gaze was measured.

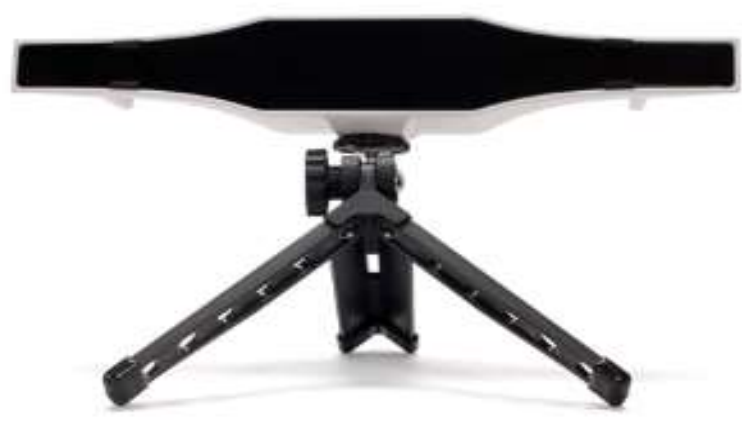

Fig. 2. Eye Tracker (Gazepoint's 'GP3')

Source: Gazepoint official website

\subsection{Experimental Stimuli}

The experimental stimuli used in this study are social robots reminiscent of human appearance and characteristics, and these robots are currently on the market or are known to the public. In order to select a robot to be used in this study among various robots, a pilot experiment was performed. In the pilot experiment, various social robots were presented to 8 college students, and among them, the robot that showed the best emotional expression was selected. However, in this study, factors related to the function of the robot were not considered. Therefore, as a result of pilot experiment focusing on the design of the robot, four types of robots were selected: 'Zenbo', 'Nao', 'Mero-S', and 'Mero-3'.

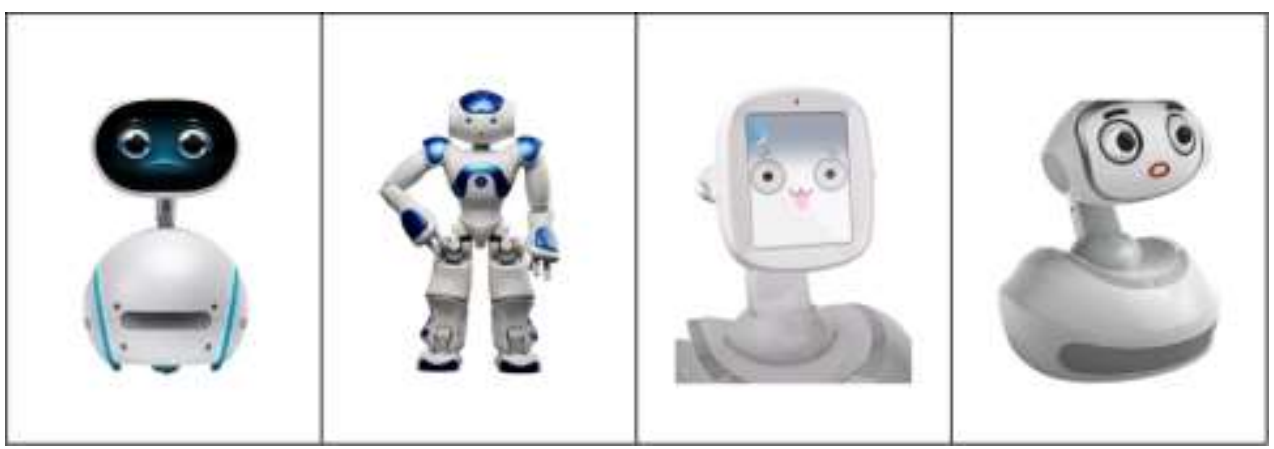

Fig. 3. Social Robot Stimuli

Source: The official website of Zenbo( $\left(1^{\text {st }}\right)$, Nao $\left(2^{\text {nd }}\right)$, Mero-S( $\left.3^{\text {rd }}\right)$, and Mero-3(Final Picture) 


\subsection{Experimental Process and Stimulus Presentation}

This study was informed to the participants before the experiment that it was an experiment related to the design evaluation of the social robot so that the experiment participants could focus on the design of the social robot. Therefore, information such as social robot function or technical level was not provided in the experimental stimulus. The specific experimental process is as follows.

The monitor on which the stimulus is presented is $1920 \mathrm{X} 1080$ size and conforms to the 24-inch standard. The distance between the participant and the monitor was about $50 \mathrm{~cm}$ to $6 \mathrm{~cm}$, and the stimuli were presented to the participants for total 10 minutes. At this time, 'Calibration' was performed to adjust the gaze of the participants before the stimulus was presented. And before the stimuli were presented, in order to control the effect of the participant's gaze on the experiment, a pre-prepared gaze point was first presented, and the starting point of the gaze was fixed. In addition, in order to control the background other than the robot presented, all backgrounds were erased and treated as white.

On the monitor, the stimulus was placed in the center so that the upper and lower and left and right intervals were the same. However, since the size of the actual social robot is different, the image size of the stimulus was adjusted to be the same. As a result, each robot occupies $25 \%$ of the area on the monitor and has a size of width $(12.5 \mathrm{~cm}) \mathrm{X}$ length $(22.5 \mathrm{~cm})$. Therefore, participants look at 4 robots on one screen for 30 seconds.

\subsection{Measurement}

The gaze responses of the participants collected through the experiment of this study were Fixation, First Visit, Total Viewed, and Revisits. Specifically, it is as follows. First, Fixation is a response to how long the participant stayed focused on a specific area. In this study, it was judged that fixation appeared when the gaze stayed for more than 0.1 seconds. Second, First Visit refers to the time it takes for the participant to first look at a specific area. Therefore, First Visit can be used as an indicator of which part of the stimulus the participant wants to see first [27]. Third, Total Viewed is a response to how long the participant's gaze stayed on a specific area. Total Viewed is known as a measurement that can grasp the overall interest of the participants and can discover key features in design [25]. Finally, Revisits refers to the number of times the gaze deviated from a specific area and then returned again. Therefore, it is possible to check the gaze path and attention level of the participant through Revisits [28].

In this study, the face, eyes, and mouth, which are external designs related to the emotional expression of social robots, were designated as main Areas of Interest (AOI). Furthermore, in order to evaluate the overall design of the social robot, the whole body of the robot was also designated as a separate AOI.

In this study, four variables were collected as variables that reflect consumer beliefs about social robots: 'attitude toward robots', 'emotional expression, 'facial prominence', and 'anthropomorphism'. Specifically, it is as follows. First, the attitude toward the robot was measured with the question 'I have a good feeling for the robot' [17]. And, as for the emotional expression, it was checked whether the participant could recognize the robot's expression with the question 'The robot's expression shows well' [29]. Facial prominence was measured as 'the robot's face stands out' [30], and anthropomorphism is a question of 'the presented robot feels like a human', which attempts to reflect the degree of anthropomorphism of the robot [31]. 


\section{Expected conclusions}

This study approached the appearance of the robot, one of the various design research flows of social robots, using eye tracking. To verify this, a research model for design evaluation of social robots was constructed by organically linking participants' gaze tracking indicators measured from various areas of interest of social robots, consumer beliefs about social robots, and consumer attitudes toward social robots. Specifically, we proposed and validated a mechanism that a user's attitude toward social robots can be formed through consumer gaze response (direct path) and consumer beliefs (indirect path). This is expected to provide specific insights related to social robot design.

The theoretical and practical implications expected through this study are as follows. As a theoretical implication, first, it can be suggested that an individual's beliefs about social robots are related to gaze response. Second, the target of the consumer's gaze response was extended to the robot's face, subdivided elements, and the entire body. Through this, more detailed and wide range of gaze response data can be reflected in the research model. Finally, the path through which consumers' attitudes toward social robots are formed can be divided into a direct path through gaze response and an indirect path through consumer beliefs.

As a practical implication, first, this study emphasizes the importance of the face, especially the eyes and lips, in the design of social robots. People's evaluation of social robots is similar to evaluating real people. Therefore, it is necessary to include factors that are considered important while evaluating the appearance of a real person in the design of the social robot appearance. Second, the attitude of consumers toward social robots is not formed only by evaluation of one factor, but is formed through consumer beliefs considering various factors in a complex way. Therefore, in the design of social robots, it is necessary to clearly define the value that the robot wants to deliver to consumers, and to derive factors suitable for the value so that the optimal design can be achieved. Lastly, it suggests the possibility that scientific experimental tools such as eye tracking can be utilized in design. Although only eye tracking was used in this study, if various measurement tools such as EEG analysis or skin conduction response are used later, it will be able to contribute to a more scientific and objective design.

\section{Acknowledgements}

This research was supported by the BK21 FOUR (Fostering Outstanding Universities for Research) funded by the Ministry of Education (MOE, Korea) and National Research Foundation of Korea (NRF).

\section{References}

1. C. Breazeal, Toward sociable robots. Robotics and autonomous systems, 42, 3-4, 167175 (2003)

2. M. Mori, Bukimi no tani [the uncanny valley]. Energy, 7, 33-35 (1970)

3. T. Fong, I. Nourbakhsh, K. Dautenhahn, A survey of socially interactive robots. Robotics and autonomous systems, 42, (3-4), 143-166 (2003)

4. F. Hegel, C. Muhl, B. Wrede, M. Hielscher-Fastabend, G. Sagerer, Understanding social robots. IEEE Trans. In 2009 Second International Conferences on Advances in Computer-Human Interactions, pp. 169-174 (2009) 
5. C. Bartneck, J. Forlizzi, A design-centred framework for social human-robot interaction. In RO-MAN 2004. 13th IEEE international workshop on robot and human interactive communication, pp. 591-594 (2004)

6. K. Kim, D. Park, Design Evaluation Mode Based on Consumer Valuesl: Three-Step Approach from Product Attributes. Perceived Attributes, to Consumer Value," Intelligence Information Research, 23(4), 57-76 (2017)

7. M. A. Salichs, Á. Castro-González, E. Salichs, E. Fernández-Rodicio, M. MarotoGómez, J. J. Gamboa-Montero, M. Malfaz, Mini: A New Social Robot for the Elderly. International Journal of Social Robotics, 1-19 (2020)

8. A. A. Ramirez-Duque, T. Bastos, M. Munera, C. A. Cifuentes, A. Frizera-Neto, RobotAssisted Intervention for children with special needs: A comparative assessment for autism screening. Robotics and Autonomous Systems, 127, 103484 (2020)

9. H. Chen, H. W. Park, C. Breazeal, Teaching and learning with children: Impact of reciprocal peer learning with a social robot on children's learning and emotive engagement. Computers and Education, 150, 103836 (2020)

10. J. Wirtz, P. G. Patterson, W. H. Kunz, T. Gruber, V. N. Lu, S. Paluch, A. Martins, Brave new world: service robots in the frontline. Journal of Service Management, 29(5) (2018)

11. G. McLean, K. Osei-Frimpong, Hey Alexa.. examine the variables influencing the use of artificial intelligent in-home voice assistants. Computers in Human Behavior, 99, 2837 (2019)

12. J. Borenstein, R. Arkin, Robotic nudges: the ethics of engineering a more socially just human being. Science and engineering ethics, 22(1), 31-46 (2016)

13. J. Borenstein, R. C. Arkin, Nudging for good: robots and the ethical appropriateness of nurturing empathy and charitable behavior. Ai and Society, 32(4), 499-507 (2017)

14. B. R. Duffy, Anthropomorphism and the social robot. Robotics and autonomous systems, 42(3-4), 177-190 (2003)

15. M. L. Walters, D. S. Syrdal, K. Dautenhahn, R. Te Boekhorst, K. L. Koay, Avoiding the uncanny valley: robot appearance, personality and consistency of behavior in an attention-seeking home scenario for a robot companion. Autonomous Robots, 24(2), 159178 (2008)

16. M. Blow, K. Dautenhahn, A. Appleby, C. L. Nehaniv, D. C. Lee, Perception of robot smiles and dimensions for human-robot interaction design. IEEE Trans. In ROMAN 2006-The 15th IEEE International Symposium on Robot and Human Interactive Communication, 469-474 (2006)

17. J. M. Park, K. S. Koo, S. S. Hong, The Study on the Proportional Preference for Humanoid Robot. Journal of Digital Design, 14(2), 725-734 (2014)

18. J. Fink, Anthropomorphism and human likeness in the design of robots and human-robot interaction. In International Conference on Social Robotics, pp. 199-208. Springer, Berlin, Heidelberg (2012)

19. B. S. Kim, S. I. Kim, User Preference for the Personification of Public Service Robot. Journal of Digital Convergence, 18(2), 361-366 (2020)

20. C. Breazeal, Emotion and sociable humanoid robots. International journal of humancomputer studies, 59(1-2), 119-155 (2003)

21. S. Kim, D. Oh, The Kinesics for emotional expression in the household social robot Focus on the head movement of the robot. The Korean Society of Science \& Art, 35, 69$82(2018)$ 
22. D. S. Park, Y. H. Pan, A Study on User's Perception and Preference Based on Emotions Expressed through The Eyes of a Social Robot. Journal of Communication Design, 67, 24-35 (2019)

23. S. Jang, H. K. Cho, Analysis of User's Eye Gaze Distribution while Interacting with a Robotic Character. Journal of Korea Robotics Society, 14(1), 74-79 (2019)

24. Y. Liu, F. Li, L. H. Tang, Z. Lan, J. Cui, O. Sourina, C. H. Chen, Detection of humanoid robot design preferences using EEG and eye tracker. IEEE Trans. In 2019 International Conference on Cyberworlds, 219-224 (2019)

25. M. Y. Choi, A Study on the Method of Eye Tracking Analysis Based on the Properties in Visual Perception of User -With Emphasis on the Development of AnalysisFramework for Product Design. Journal of Korean Society of design science, 16(4), 197$206(2003)$

26. E. S. Seo, The Suggestion for the Design of Eye Tracker to Promote the Study on the Gaze Tracking Interface. Journal of Cultural Product and Design, 50, 145-152 (2017)

27. R. J. Jacob, and K. S. Karn, Eye tracking in human-computer interaction and usability research: Ready to deliver the promises. In The mind's eye, 573-605 (2003)

28. J. H. Kim, J. Y, Kim, Characteristics of Visual Perception for the Bluetooth Headset Image Based on Eye-Tracking Experiment. Journal of The Korean Society of Living Environmental System, 24(3), 433-445 (2017)

29. S. W. Jeong, Designing Facial Expressions of an Educational Assistant Robot by Contextual Methods. Archives of Design Research, 26(2), 409-435 (2013)

30. Y. J. Oh, Y. S. Shin, J. H. Lee, J. W. Kim, A Study on Interaction Design of Companion Robots Based on Emotional State. Journal of Digital Contents Society, 18(7), 1293-1301 (2017)

31. D. Kim, S. Choi,The Effect of Expressions of Self-consciousness in Social Robots during Social Attraction. Journal of KIISE, 46(7), 653-663 (2019) 